\title{
DIAGNOSTIC ASPECTS OF LEUKOPLAKIA
}

\author{
H.P. Kamilov., A.A. Kadyrbaeva, N.O. Asilbekova. \\ Tashkent State Dental Institute, Tashkent
}

Leukoplakia of the oral mucosa is an actual problem of modern dentistry due to the fact that the number of patients is growing every year, and the results of treatment can not be considered satisfactory. This disease of the MMOC is characterized by a persistent course and in $20-30 \%$ of cases is subjected to malignancy. Long-term conservative treatment often does not guarantee patients full recovery, and the surgical methods used are not always possible due to the complexity of the anatomical structure of the oral cavity and the prevalence of the pathological process. (C.Aquilina et all - 2003; M.Bocor-Bratic-2003; I.Ishii et all - 2003; V.Prass et all - 2005) The frequency of relapses according to some authors varies from 7 to $\quad 40 \%$ (J.Ishii et all-2003). The aim of the study was to investigate the various methods of diagnosis of individual forms of leukoplakia of oral mucosa, taking into account the characteristics of the clinical and morphological manifestations of the disease. Materials and methods of the research. We examined 27 patients (16 men, 11 women) aged 48 years old, suffering from various forms of leukoplakia, who applied to the Department of Therapeutic Dentistry of the clinic of the Tashkent State Dental Institute. Conducted clinical and laboratory examination methods. Anamnesis of the disease was studied, the state of the oral mucosa, the duration of the disease, its shape, size and location were determined. From laboratory studies used cytological or histological studies, through the use of light microscopy. The morphological study of the material made it possible to obtain information not only about the structural features of leukoplakia, but also contributed to the establishment in the epithelial tissue of cellular and nuclear polymorphism, the degree of chromatin staining intensity, the determination of the nuclearcytoplasmic ratio, and mitotic activity. Results of the research. For define of forms of leukoplakia we used classification, proposed by Mashkileyson. Flat or simple form of leukoplakia was diagnosed in $63,3 \%$, verrucous form - in $27,5 \%$ and erosive form - in 9,2\% cases. Detailed study distribution patients on age and gender with regard to clinical manifestations disease shows about volume, what simple form of leukoplakia most often taped aged from 20 to 40 years, in that time, as verrucous and erosive - prevail among men after 40 years. Study distribution patients with regard to localization disease oral mucosa revealed, what most often leukoplakia determined in areas of cheeks and several less often - in areas fabrics bottom cavity mouth, language and bottom lips. Distribution of various forms of leukoplakia on individual anatomical sites oral mucosa revealed, that flat form of the disease equally often strikes all sites cavity mouth, whereas verrucous and erosive form, usually, is localized in areas of cheeks and language. Study of anamnesis data, previous appearance of leukoplakia of oral mucosa, gave possibility to install that at $26,2 \%$ patients identified with first signsof the disease who noticed presence of chronic injuries oral mucosa. In 
$39,2 \%$ cases had place excessive use of nas and smoking. From general number surveyed patients, in $47,6 \%$ case were marked following comorbidity: chronic disease of gastrointestinal tract, warmly-vascular violations, endocrine disease and so on. First symptoms of the disease were white plaques on oral mucosa, surrounded by thin hyperemic ring, also patients always noticed the feeling of burning. Clinically at simple form of leykoplakia of oral cavity was determined by presence of white spots in form of limited sites. They, not towered over surrounding cloth and had various shades. At verrucous form of leykoplakia affected plot reminded dense greyish color plaque, surface which was bumpy, but in individual cases - fold, there were determined picture of expressed hyperkeratosis. Usually, crack and erosion at flat and errucous forms practically were not found, in that time as they were integral part of erosive form of leykoplakia. For cytological research of erosive surface take smear-imprint on substantive glass. Smear stained by Papanikolau. Histological study of simple form of leykoplakia revealed, that disease maybe characterized, as hyperplastic, chronic inflammation, in which identifies sites of metaplasy. At this, in all cases of hyperplastic process, as and methaplasy epithelium, constantly it was accompanied by expressed process of keratinization, with alternation hyper- and parakeratosis zones. Acanthosis of cells was emphasized in those cases, when keratinization bore nature of parakeratosis. Inflammatory reaction in sub tissues usually not determined. Picture clearly was presented at verrucous form. At this, grainy layer mucosal shell consisted from significant number series cells. Especially often grainy layer was emphasized in those places, where was determined by hyperkeratosis. Here cells contained large grain keratohyaline. In individual cases celebrated pronounced acanthosis, which it was accompanied significant elongation and extension epithelial outgrowths. In connective tissue stroma affected sites mucosal оболоски determined diffuse chronic inflammation with expressed лимфоплазмоцитарной reaction. In individual cases spike layer was submitted by several layers of cells, quantity which in individual cases reached 810 series. Cells were different values and form, they weakly were dyed, their kernel were large and hyperchromatic. Not often there were noted presence in nuclei 2-3 nucleouluses and row violation of cells location. Rarely are identified atypical mitosis. In erosive form of leykoplakia, also noted thickening of epithelial layer, basically for account of increase of basal layer. In some cases epithelial strands deeply penetrated in connecting tissues, on circle which had place round cell infiltration. It is necessary to note that discomlectation of spiky cells layer and cellular atypia especially was expressed at erosive form of leykoplakia. Here inflammatory reaction in stroma reached maximum, whereas at simple form of leykoplakia, inflammatory reaction was expressed considerably less. So, received by us data show, that at all forms of leykoplakia determined significant degenerative changes in basal layer and phenomenon of acanthosis. However, appearance of long epithelial cords, with expressed atypia of cells points on tendency to malignant process. Clinically at these patients in areas of pathological focus were noted presence of erosion and expression of expressed pain syndrome. On circle of leykoplakia it was palpated tight infiltration, but on the center - anchor 
elements. This picture is revealed at flat form of leykoplakia in $5,2 \%$, at verrucous - $18,7 \%$ and of erosive form - in 37,5\% cases.

Conclusions. Analysis of background processes preceding the occurrence of oral mucosa cancer showed that certain forms of leukoplakia transform into a malignant process and are represented by a highly differentiated form. An in-depth study of the clinical and morphological manifestations of leukoplakia of the oral mucosa showed that the initial signs of malignancy in a simple (flat) form of leukoplakia are detected only in $5.2 \%$, in case of verrucosis in $18.7 \%$, and in erosive cases $-37.5 \%$ of cases. This picture is mainly observed in male patients, with duration of leukoplakia of more than 6 months, who have a anamnesis of chronic injuries of the mucous membrane, are heavy smokers and drink alcohol intensively.

\section{Bibliography}

1. Features of the manifestation and methods of diagnosis of precancerous lesions of the oral mucosa. Abdullakhodzhayeva MS, Kamilov Kh.P., Kadyrbaeva AA, Asilbekova N. / / Stomatologiya № 4, 2017. - from 18-19 2. Endocrine and Immune Systems in the Organism. E. Xvedelidze, N. Rigishvili, M. Rukhadze, L. Zurabashvili. // Bull. of Georgian Acad. Of Sci., 2006, n. 173, \# 2 ,

3. Al-Hashimi, I. Advances in the Diagnosis and Management of Oral Diseases / I. Al-Hashimi // The Open Pathology Journal. - 2011. - Vol. 5. - P. 1-4. 4. Alteri, R. Cancer Facts \& Figures 2013 / R. Alteri et al. - Atlanta: American Cancer Society, 2013. - 64 p. 